\title{
Laboreal
}

Volume $3 \mathrm{~N}^{\circ} 2$ | 2007

Trabalho infantil

\section{Las disponibilidades de tiempo en la construcción de las normas temporales del trabajo}

As disponibilidades de tempo na construção de normas temporais de trabalho Les disponibilités de temps dans la construction des normes temporelles de travail

Time availabilities in the construction of work temporal norms

\section{Esteban Martinez}

\section{(2) OpenEdition}

\section{Journals}

Edición electrónica

URL: http://journals.openedition.org/laboreal/12645

DOI: $10.4000 /$ laboreal. 12645

ISSN: 1646-5237

\section{Editor}

Universidade do Porto

\section{Referencia electrónica}

Esteban Martinez, «Las disponibilidades de tiempo en la construcción de las normas temporales del trabajo », Laboreal [En línea], Volume 3 №2 | 2007, Publicado el 01 diciembre 2007, consultado el 24 septiembre 2020. URL : http://journals.openedition.org/laboreal/12645 ; DOI : https://doi.org/ 10.4000/laboreal. 12645

Este documento fue generado automáticamente el 24 septiembre 2020.

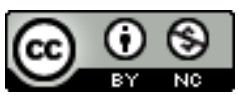

Laboreal está licenciado com uma Licença Creative Commons - Atribuição-NãoComercial 4.0 Internacional. 


\section{Las disponibilidades de tiempo en la construcción de las normas temporales del trabajo}

As disponibilidades de tempo na construção de normas temporais de trabalho Les disponibilités de temps dans la construction des normes temporelles de travail

Time availabilities in the construction of work temporal norms

\section{Esteban Martinez}

\section{REFERENCIA}

Martinez, E. (2007). Les disponibilités de temps dans la construction des normes temporelles de travail. Doctorat en Sciences politiques et sociales, Université Libre de Bruxelles, Bruxelles.

\section{NOTA DEL EDITOR}

Manuscrito recibido en : noviembre/2007

Aceptado tras peritage en : diciembre/2007

\section{Introducción}

1 Las transformaciones contemporáneas del tiempo de trabajo se aprecian tomando como punto de referencia la norma temporal fordista que se consolidó, en el contexto industrial, como una combinación compleja entre formas de estabilización de la mano de obra, formas de delimitación del espacio y del tiempo de trabajo y formas de racionalización tayloriana de la producción. En ese contexto, el tiempo de trabajo 
estaba estrictamente medido y delimitado. De esta manera Pierre Naville (1972) subrayaba la importancia clave de la dimensión del tiempo en el análisis económico y social ya que, en la sociedad salarial, el tiempo se divide esencialmente en tiempo de trabajo y tiempo de no-trabajo.

2 Pero la relación con respecto al tiempo ha cambiado durante las últimas décadas, dado que el trabajo se ha hecho mas complejo, los intereses de los empleadores se han diversificado en un contexto de mutación económica y de búsqueda de mayor flexibilidad, y la composición del asalariado se ha modificado, con el aumento de las cualificaciones y del empleo femenino. En la actualidad, se rechaza la categoría del tiempo en su función de medida y de evaluación del trabajo, pero simultáneamente interviene más que nunca en la coordinación del trabajo y de la vida social. Desde este punto de vista, parece conveniente introducir en el análisis el concepto de disponibilidad temporal ya que los esfuerzos de sincronización de la actividad productiva y los procesos de diferenciación del asalariado se basan hoy en día más en formas de disponibilidad temporal que en marcos temporales formales.

3 La óptica de las disponibilidades de tiempo permite en efecto sobrepasar, al englobarlo, el problema de la medida del trabajo por el tiempo, dado que la participación de los trabajadores parece menos regida que antes por los límites de la duración del trabajo, en un contexto en el que el resultado prevalece sobre el cálculo de las horas o en el que ell tiempo llega a ser intercambiable y el horario irregular. Dicho de otro modo, que se cuente o no el tiempo de trabajo, la eficacia de la organización se apoya de todas maneras en la disponibilidad temporal y en la implicación subjetiva de los trabajadores. Esta óptica conduce asimismo a trasladar el enfoque de la lógica de las organizaciones hacia las preocupaciones de los asalariados, que deben enfrentarse al reto que plantea en la vida cotidiana la articulación de una pluralidad de tiempos. La feminización del empleo constituye aquí un hecho social de gran importancia, capaz de modificar la relación con respecto al tiempo, tanto en el espacio público como en el privado. La participación creciente de las mujeres en la actividad profesional pone en evidencia la presión que ejercen también los tiempos de la vida privada, que permanecieron durante mucho tiempo a la sombra del trabajo, cuando en un universo laboral masculino, el tiempo fuera del trabajo podía considerarse como un tiempo libre, un tiempo de ocio ganado al trabajo (Adam, 1995).

4 Al estudiar las relaciones entre los marcos temporales resultantes de las formas actuales de organización del trabajo y la experiencia temporal de los trabajadores enfrentados a una pluralidad de tiempos sociales, el objetivo es demostrar, siguiendo a Mateo Alaluf (2000), que las formas de disponibilidad temporal y de aceptabilidad de las condiciones laborales son cada día más determinantes en el funcionamiento del mercado del trabajo y en la división social y sexuada del empleo.

\section{Metodología}

5 La investigación se ha centrado en tres grupos profesionales muy diferentes desde el punto de vista de la cualificación, de la posición social y del género : mandos y técnicos del banco, enfermeras de hospital y operarios/as de limpieza. Cada una de las monografías combina datos jurídicos, estadísticos y cualitativos. En una primera aproximación, las transformaciones del tiempo de trabajo se perciben en el desarrollo de una reglamentación permisiva. El análisis de los convenios colectivos pone de relieve 
el proceso de diversificación de la organización temporal del trabajo. Pero, a diferencia de los tiempos de trabajo formales, las formas concretas de disponibilidad temporal dejan pocas huellas en la codificación jurídica o estadística. Por lo tanto, hemos privilegiado un enfoque metodológico comprensivo, capaz de poner en evidencia las representaciones del tiempo y las interrelaciones entre los tiempos dentro y fuera del trabajo. En esta investigación se ha tratado de poner en relación el marco temporal instituido a través de la negociación colectiva, las representaciones del tiempo vehiculadas por los actores sociales de la regulación y la experiencia del tiempo vivida por los trabajadores. Se ha procurado poner de relieve los marcos temporales de referencia partiendo del análisis de los acuerdos sociales, comentados por los representantes sindicales y empresariales. El análisis de las representaciones personales del tiempo se ha basado en entrevistas en profundidad con una muestra no representativa de trabajadores de distintas empresas y pertenecientes a cada unos de los grupos profesionales estudiados. En las entrevistas se abordaron las representaciones y usos del tiempo, las tensiones o convergencias que aparecen entre los tiempos laborales y los otros aspectos de la vida.

6 Este enfoque ha permitido destacar diversas modalidades de flexibilización del trabajo y, según la tipología de Javier Callejo (2004), los impulsos sociales de la disponibilidad temporal, articulados en torno a la profesión, a la carrera o a la preservación del empleo.

\section{Enfermeras de hospital}

7 Las enfermeras están expuestas a horarios de trabajo variables e irregulares en relación con las temporalidades sociales comunes. Poniendo de lado la perspectiva vocacional que se ha atribuido al trabajo de las enfermeras, se puede constatar que su tolerancia con respecto a horarios incómodos, poco compatibles con las cargas familiares, se basa en una implicación positiva con la profesión y en la aceptación de las imposiciones objetivas de esta actividad. En este sentido, la disponibilidad temporal de las enfermeras puede calificarse como "profesional », pues el motor de su implicación resulta ser la interiorización de las exigencias de la profesión y la adhesión a los valores que conllevan.

8 El reto para los gerentes del hospital es combinar el imperativo financiero con la necesidad de evitar conflictos con las enfermeras en el marco de un mercado de trabajo tenso. En este contexto, las obligaciones de la vida familiar se reconocen y legitiman hasta cierto punto, mediante la planificación de los horarios, la sustitución de las enfermeras en reposo compensatorio o dejando a las enfermeras la posibilidad de arreglarse entre ellas. Pero esta organización colectiva no basta para solucionar las dificultades que encuentran las enfermeras en la gestión de la vida diaria. Al fin y al cabo, las enfermeras logran limitar los periodos de dificultad a través de compromisos individuales, pasando a un régimen de trabajo a tiempo parcial o recurriendo al trabajo temporal. 


\section{Mandos y técnicos del banco}

9 Los mandos y técnicos del banco forman un grupo profesional heterogéneo con contornos vagos. Sus funciones se sitúan entre las del empleado administrativo y las del directivo. La disponibilidad temporal se concretiza principalmente en este caso en la elasticidad de fin de jornada que los llevan a acumular horas extras con respecto al horario normal de trabajo. Sin embargo, el hecho de que dispongan de cierto margen de decisión y de que las horas extras no excedan ciertos límites considerados como aceptables atenúa las tensiones entre la vida laboral y la vida privada.

10 La aceptación de duraciones de trabajo elevadas remite a las representaciones sociales del estatuto, como " asalariado de confianza ». Tener que responder a obligaciones de resultado y por ello no tener que contar su tiempo es una manera de distinguirse. La disponibilidad se valoriza en la medida en que es la expresión del grupo con el que se identifica o al cual se quiere pertenecer. En este sentido, la disponibilidad de los cuadros puede calificarse de "corporativa». Las principales motivaciones son la posición en la organización y el desarrollo de la carrera. Pero en los usos del tiempo, la definición de los contornos de la categoría profesional y el género son variables determinantes.

11 Así, fuera de los directivos, los mandos medios y técnicos son considerados sólo como empleados que deben respectar el reglamento del tiempo de trabajo. Pero su discurso refleja muy bien la distancia que se marca entre la aplicación de la reglamentación y los usos del tiempo. Hasta cierto punto, las mujeres pueden adoptar una conducta diferente, trabajar a tiempo parcial, atenerse a horarios más regulares, sin renunciar a una carrera. Pero, la disponibilidad que se espera de los mandos medios y técnicos se convierte en un factor de exclusión a la hora de ascender jerárquicamente, donde se impone el modelo masculino. La exigencia de disponibilidad temporal se agrega entonces a otros procesos selectivos para limitar el acceso de las mujeres a las posiciones superiores.

\section{Operarios/as de la limpieza}

12 En este caso, las relaciones sociales de la subcontratación determinan el conjunto de las condiciones de trabajo. Las tareas de limpieza se realizan en los locales del cliente. Por eso, el cliente asume una parte de las responsabilidades empresariales. Determina, en particular, los tiempos de trabajo efectivos. Ahora bien, las tareas de limpieza se organizan según un horario discontinuo con secuencias de trabajo situadas temprano por la mañana y tarde por la noche. Así pues, los operarios de la limpieza padecen doblemente el tiempo de trabajo. Se ven obligados al tiempo parcial y por ello a un salario parcial. A la debilidad del salario se agrega el sometimiento a horarios diarios incómodos. A estos elementos hay que agregar la violencia a nivel de las relaciones de trabajo : en un contexto marcado por la existencia de un conflicto permanente en torno al problema de la duración y del horario de trabajo, se instaura la disciplina por medio de la amenaza del paro. El aviso que anuncia un posible despido se convierte en el medio de comunicación entre la jerarquía y los trabajadores.

13 La tolerancia de los operarios de la limpieza frente a las malas condiciones de trabajo se explica porque éstos tratan de "ganar horas", ya sea prestando horas extras, ya sea 
sumando varios empleos. En este sentido, la disponibilidad temporal proviene de una lógica de subsistencia económica. Preservar el empleo es lo que constituye el motor.

La dimensión de género instala otra línea de división entre los trabajadores del sector. La división sexual del tiempo de trabajo conlleva la división de tareas y las representaciones de los papeles masculino y femenino en la familia y en el trabajo. Incluso si al principio ambos están sometidos a obligaciones temporales comparables, las desigualdades son palpables. Los varones ocupan más a menudo empleos a tiempo completo mientras que las mujeres deben contentarse con las "migajas de empleo ", según la expresión de Tania Angeloff (1999).

\section{Conclusiones}

Que salga de acuerdos formales o de acuerdos sociales implícitos, la disponibilidad temporal puede percibirse como un esfuerzo de coordinación temporal concedido por los trabajadores en sistemas de empleo marcados por la flexibilidad de la organización del trabajo y la transformación de las formas de subordinación salarial. Desde este punto de vista, las exigencias de disponibilidad temporal contribuyen a la transformación de la norma social del empleo que se había consolidado en la postguerra y que implicaba una mayor regularidad de los tiempos de trabajo y una fuerte sincronización de los tiempos sociales (Prieto, 1999). Sin embargo, cabe interrogarse sobre la aprehensión del tiempo de trabajo como condición de trabajo, es decir como objeto de la negociación colectiva. Lo cual, hoy en día plantea grandes desafíos en un contexto en el que los arreglos temporales implícitos se apartan de los marcos formales o en el que la organización del trabajo ya no es algo que se construye en la empresa sino que parece imponerse por exigencias del mercado.

Respecto a los procesos de construcción de las normas temporales del trabajo, no presenciamos el reemplazo de une norma temporal "estándar " basada en empleos estables, a tiempo completo y con horarios colectivos, por otra que sea flexible, que se pueda caracterizar de manera unívoca por la inestabilidad del empleo, el trabajo a tiempo parcial y horarios individualizados. Se ve que las temporalidades del trabajo se inscriben en relaciones sociales de trabajo diferenciadas y desiguales. Así, la manera diferenciada de reconocer la disponibilidad temporal de los trabajadores contribuye a la segmentación del mercado laboral. Mientras que esta se toma en cuenta implícitamente en el caso de los mandos o técnicos que benefician de compensaciones simbólicas y materiales, se ignoran ampliamente en el caso de la subcontratación, cuando la aceptación de horarios y jornadas de trabajo atípicas condiciona el acceso al empleo. Igualmente, en el contexto de un reparto desigual del trabajo doméstico y parental, si la disponibilidad con respecto a la familia se toma en cuenta en el caso de las enfermeras, dando lugar a revisiones de la organización del trabajo, esta es ignorada en el caso de las directivas del banco o de las operarias de limpieza.

17 Las variantes que toma la norma temporal flexible emergente reflejan las divisiones sociales y sexuales del empleo. Así, tener en cuenta las disponibilidades de tiempo permite profundizar el análisis de la segmentación del mercado del trabajo, al distinguir un régimen flexible autónomo, atribuido a los directivos, y un régimen flexible heterónomo, caracterizado por el trabajo a tiempo parcial impuesto (Bouffartigue \& Bouteiller, 2003) o por estrategias de reducción de costes salariales 
basadas en la desconexión parcial del tiempo y del salario, o sea en un calculo estricto del tiempo de trabajo (Rubery, Ward, Grimshaw \& Beynon, 2005).

\section{BIBLIOGRAFÍA}

Adam, B. (1995). Time Watch. The Social Analysis of Time. Cambridge : Polity Press.

Alaluf, M. (2000). Le travail du temps. In G. de Terssac et D.-G. Tremblay (sous la direction de) (204-216), Où va le temps de travail ? Toulouse : Octarès.

Angeloff, T. (1999). Des miettes d'emploi : temps partiel et pauvreté. Travail, Genre et Sociétés, 1 , 43-70.

Bouffartigue, P.\& Bouteiller, J. (2003). À propos des normes du temps de travail. De l'érosion de la norme fordienne aux normes émergentes. Revue de l'IRES, 42, 1-23.

Callejo, J. (2004). Disponibilidad temporal corporativa y género : aproximaciones empiricas, Anduli. Revista Andaluza de Ciencias Sociales, 4, 31-60.

Naville, P. (1972). Temps et technique. Les structures de la vie de travail. Genève, Paris : Librairie Droz.

Prieto, C. (1999) (ed.). La crisis del empleo en Europa. Germania : Alzira Rubery, J., Ward, K., Grimshaw, D. \& Beynon, H. (2005). Working Time, Industrial Relations and the Employment Relationship. Time and Society, 15, 1, 89-111.

\section{AUTOR}

\section{ESTEBAN MARTINEZ}

Université Libre de Bruxelles - METICES 44, Avenue Jeanne - B 1050 Bruxelles Belgique emartin@ulb.ac.be 